\title{
Géométrie interne du Christ portant la croix
}

\section{Internal geometry of Christ carrying the cross}

\author{
Jean-Pierre Crettez ${ }^{1}$ \\ ${ }^{1}$ Chercheur émérite à Telecom Paris
}

RÉSUMÉ. L'étude présentée ici concerne la recherche de la géométrie interne du Christ portant la croix. Cette démarche s'appuie sur la méthodologie que nous avons développée précédemment ${ }^{1}$ et en confirme la pertinence. Elle met en évidence une action constructive voisine de celles développées par Léonard de Vinci.

ABSTRACT. The study presented here concerns the search for the internal geometry of Christ carrying the cross. This approach is based on the methodology we have developed previously ${ }^{2}$ and confirms its relevance. It highlights a constructive action similar to those developed by Leonardo da Vinci.

MOTS-CLÉS. Architecture, construction interne, géométrie interne, géométrie secrète, maillage harmonique, poncif, formes consonantes visuelles, stylisation des formes.

KEYWORDS. Architecture, internal construction, internal geometry, secret geometry, harmonic mesh, visual consonant forms, stylization of forms.

\section{Introduction : la géométrie interne}

Grâce à leur culture géométrique, les peintres de la Renaissance établissaient leur composition à l'aide de la géométrie interne qui leur permettait de dresser les lignes directrices, d'établir les relations entre les éléments picturaux, de tracer leur forme stylisée, et de mettre en place les formes consonantes visuelles.

Cependant, la géométrie interne n'est pas tracée directement sur la fresque ou sur la toile, mais de préférence sur un patron. Sur ce patron, les peintres commencent par tisser une trame géométrique régulière que nous avons appelée maillage. Le maillage carré ou le plus souvent harmonique sert de support au tracé de la géométrie interne. Lorsque l'étude de la composition est achevée, il est alors inutile de transférer le maillage et les lignes de construction, seuls les contours des formes sont transférés sur la toile ou sur le bois par la méthode du poncif. Les contours des formes tracées sur le patron, sont percés de petits trous. Le passage d'une ponce à travers ces trous reproduit simplement les contours en pointillé sur la toile. Par extension, certains appellent poncifs ces patrons percés de petits trous. Les poncifs permettent au maitre ou à ses disciples d'effectuer des répliques.

C'est pourquoi le tracé du maillage et celui de la géométrie interne n'apparaissent pas dans l'œuvre peinte, ni dans son image radiographique, et ni non plus dans son image infra-rouge : la géométrie interne demeure secrète. Mais, lorsque celle-ci a été tracée en se référant aux nœuds du maillage harmonique, les éléments picturaux issus de la géométrie interne sont quantifiés, la géométrie interne devient discrète. Détecter la géométrie interne, c'est retrouver dans le non-visible, les éléments de construction du visible ${ }^{2}$.

Inversement, lorsqu'une œuvre a été créée par un peintre sur un maillage carré ou harmonique, le caractère discret de la géométrie interne rend possible et crédible la détection des points particuliers, des éléments linéaires, et des courbes particulières comme les formes elliptiques. Il permet de déceler le maillage avec précision. Grâce à ce dernier, il devient possible de détecter les autres

\footnotetext{
${ }^{1}$ J.-P. Crettez: [3]

${ }^{2}$ J.-P. Crettez: [4]
} 
éléments picturaux, de retrouver la géométrie interne de la composition et ainsi de mettre en évidence la démarche créatrice du peintre. ${ }^{3}$ »

\section{Présentation du Christ portant la croix}

À la Renaissance apparaît l'art du portrait, en particulier le portrait vu de trois-quarts, cherchant à montrer le tempérament du modèle, et en particulier sa vie intérieure. « Tu ne feras jamais les têtes droites sur les épaules, mais tournées de côté, à droite ou à gauche..., parce qu'il est nécessaire de faire en sorte que leurs mouvements aient l'air d'être vivants et non figés. $\gg{ }^{4}$ Cette évolution du portrait a conduit les artistes à proposer une nouvelle représentation du Christ portant la croix.

S. Kourtchevsky ${ }^{5}$ a répertorié plusieurs versions du Christ portant la croix, six sont attribuées à Giampietrino, elles sont conservées respectivement : à la National Gallery de Londres, au Musée des Beaux-Arts de Budapest, au Musée Royal de Turin, au Musée des Beaux-Arts de Vienne, au Musée Diocésain de Milan et au Musée National du Château Saint-Ange de Rome ; enfin une version attribuée à Solario est conservée à la Gemäldegalerie de Berlin. Giampietrino et Solario sont des disciples de Léonard de Vinci.

La ressemblance étroite entre ces versions a conduit les historiens d'art Pietro C. Marani et Carlo Pedretti à supposer l'existence d'une composition perdue d'un Christ portant la croix. Kenneth Clark remarque dans Le Christ portant la croix de Giorgione, une imitation évidente d'un dessin de Léonard. "Ce dessin nous est connu dans ses grandes lignes par les copies qu'en firent ses élèves à Milan, à partir d'un carton aujourd 'hui perdu. $\|^{6}$

\section{Christ portant la croix (collection privée)}

À ces versions, il faut ajouter la version d'une collection privée qui a été étudiée récemment par S. Kourtchevsky ${ }^{7}$ (figure 1). C'est une peinture à l'huile sur un panneau constitué d'une seule planche de peuplier qui a pour dimensions : $60,1 \mathrm{~cm}$ de hauteur et $45,6 \mathrm{~cm}$ de largeur.

En 1969, la radiographie a dévoilé la présence d'un second personnage ${ }^{8}$ situé sous une couche de peinture noire qui a été enlevée (figure 1).

En 1991, un examen de la couche picturale et des pigments utilisés de ce tableau par l'institut ISEA de Zurich, a permis de le dater du $15^{\text {ème }}$ ou début du $16^{\text {ème }}$ siècle. En 2010 l'examen au carbone 14 par les laboratoires de Zurich et de Lyon, confirme cette datation.

Cette version n'a pas encore reçu d'attribution.

Pour retrouver la géométrie interne du Christ portant la croix, nous avons choisi cette version qui comme nous le verrons est très proche de la version de la National Gallery.

\footnotetext{
${ }^{3}$ J.-P. Crettez: [5]

${ }^{4}$ Léonard de Vinci [7]

${ }^{5}$ S. Kourtchevsky : "Christ portant la croix de la Renaissance italienne, les clefs d'une énigme ? " communication privée.

${ }^{6}$ Kenneth Clark [2]

${ }^{7}$ S. Kourtchevsky. Ibidem.

${ }^{8}$ S'agit-il de l'apôtre Jean, frère de l'apôtre Jacques, ici peint sous les traits de Salaï (disciple de Léonard) ?
} 


\section{Description du Christ portant la croix (collection privée)}

Le Christ est représenté de profil, la tête tournée de trois-quarts vers le spectateur. Il porte la lourde croix de bois (l'instrument de son supplice). Son dos en grande partie dénudé dévoile sa musculature, mais nous montre peu de traces de la flagellation.

À l'exception du personnage de droite (qui ne fait pas l'objet de cette analyse), le fond n'apporte aucune information. Le peintre n'a brossé que le Christ, seul, silencieux, enfermé dans sa souffrance comme le montre son regard d'une intensité insoutenable.

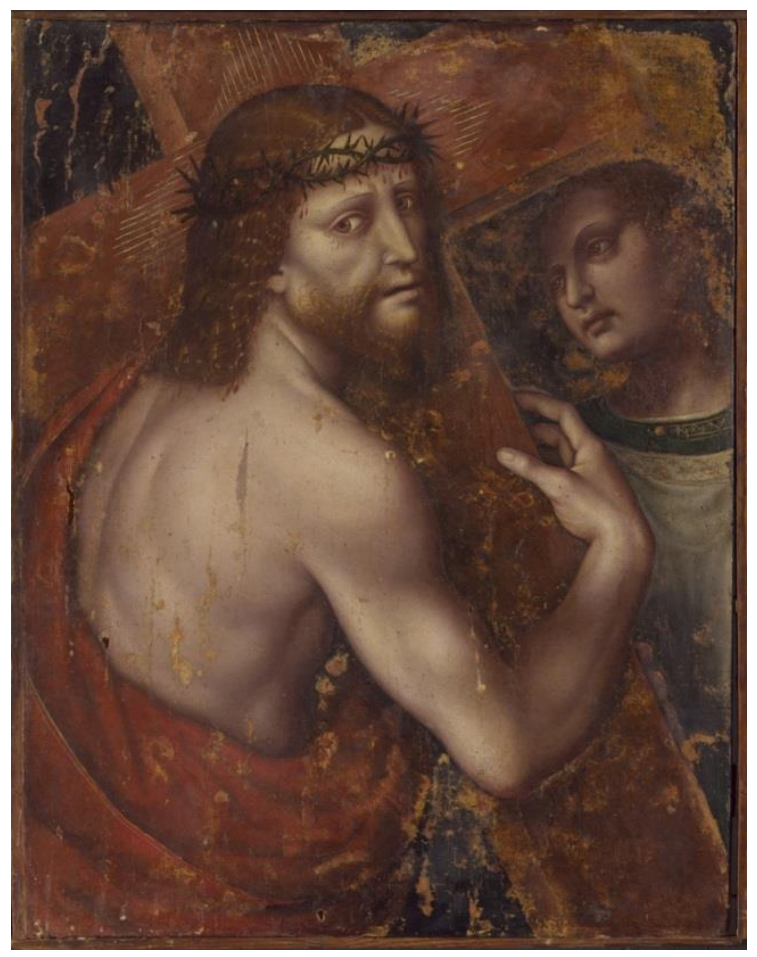

Figure 1. Le Christ portant la croix (collection privée)

\section{Recherche de la géométrie interne}

Comme on peut l'observer, la scène a été tronquée. En haut, la croix n'est pas entièrement représentée ; en bas à gauche la tunique du Christ est incomplète. Contrairement à ce que préconise Charles Bouleau', il n'est donc pas possible de se fier au cadre du tableau pour déterminer la géométrie interne. On ne peut que s'appuyer sur des éléments caractéristiques présents dans l'œuvre elle-même. Deux éléments picturaux nous semblent essentiels : les éléments linéaires délimitant les bords de la croix, la forme apparente de la tête du Christ.

\section{Les bords des bois de la croix}

Le Christ tient la croix à deux mains en l'appuyant sur son épaule gauche. Pour assurer la stabilité, il maintient la croix inclinée d'un angle $\boldsymbol{\alpha}$ (figure 2). La mesure de cet angle d'inclinaison $\boldsymbol{\alpha}$ est sensiblement égale à $64^{\circ} 70$ par rapport à la direction horizontale. Pourquoi l'auteur a-t-il choisi cet angle singulier qui à l'époque de la Renaissance, n'a pas de signification ? Probablement parce qu'à cette époque les peintres établissaient leur composition sur un maillage harmonique ${ }^{10}$. La ligne droite représentant le bord du montant de la croix a été tracée directement en passant par les nœuds

\footnotetext{
${ }^{9}$ Charles Bouleau : [1]

10 J.-P. Crettez: [3], §. 2.5
} 
d'un maillage harmonique vertical. En effet, cette droite correspond, dans un maillage harmonique vertical, à la diagonale d'un bloc vertical de deux fois trois mailles (figure 3). Le segment de droite $^{11}$ correspondant à cette diagonale est incliné d'un angle $\boldsymbol{\alpha}$ tel que tg $\boldsymbol{\alpha}=3 \sqrt{2} / 2$, soit $\boldsymbol{\alpha}=64^{\circ} 76$.

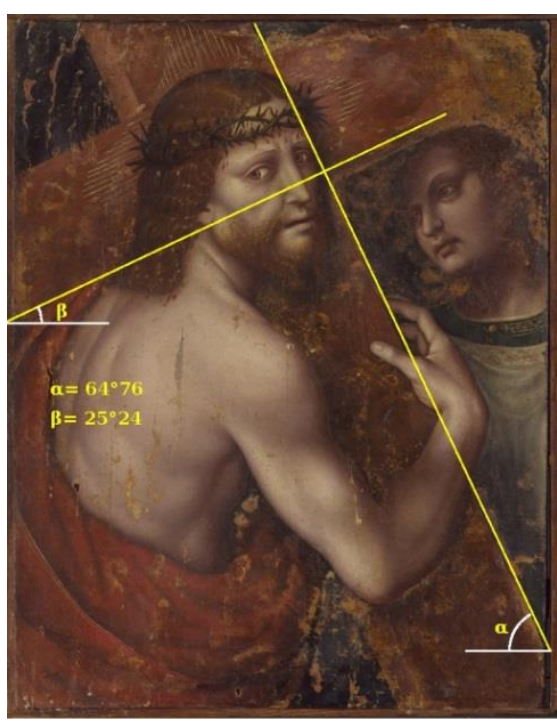

Figure 2. Angles d'inclinaison des bords de la croix passant par les nœuds du maillage

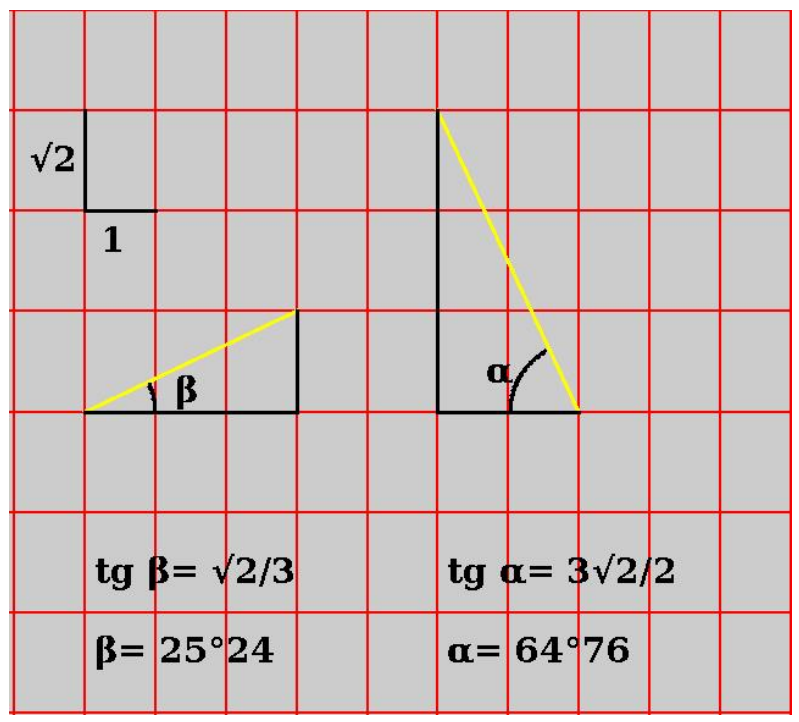

Figure 3. Maillage harmonique. Droites des bords de la croix

De même, la ligne droite représentant le bord des deux bras de la croix a été tracée directement en passant par les nœuds du même maillage harmonique vertical, elle correspond à la diagonale d'un bloc horizontal de trois mailles. Ce segment de droite est incliné d'un angle $\boldsymbol{\beta}$ tel que $\operatorname{tg} \boldsymbol{\beta}=\sqrt{ } 2 / 3$, soit $\boldsymbol{\beta}=25^{\circ} 24$. Ces deux segments de droite représentant respectivement le bord du montant et celui du bras de la croix sont orthogonaux puisque : $\operatorname{tg} \boldsymbol{\alpha} . \operatorname{tg} \boldsymbol{\beta}=1$, et par suite les bras de la croix sont bien tracés perpendiculairement au montant.

\section{Remarque :}

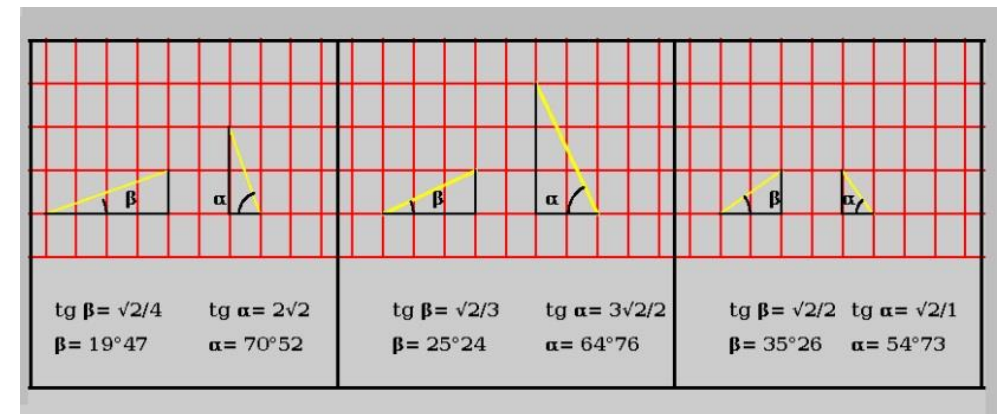

Figure 4. Couples de segments de droite orthogonaux tracés dans un petit maillage harmonique

Dans un petit maillage harmonique, les couples de segments de droite orthogonaux qui peuvent être tracés sont peu nombreux. Seuls quelques peintres géomètres devaient les connaître. La seule inclinaison $\boldsymbol{\alpha}$ qui peut être tracée entre $56^{\circ}$ et $70^{\circ}$ est précisément celle choisie par 1'auteur : $64^{\circ} 76$.

\footnotetext{
${ }^{11}$ Dans un maillage harmonique, le nombre de nœuds est fini. Le nombre de droites que l'on peut tracer en passant par les nœuds de ce maillage est fini. Leurs orientations sont "discrètes".
} 
Cette mise en évidence du processus du tracé des bois de la croix montre que cette version du Christ portant la croix possède une géométrie interne et que cette dernière repose sur un maillage harmonique vertical.

Ainsi, cette géométrie interne a permis d'établir la composition de cette œuvre sur un patron qui percé de petits trous, a formé un poncif. Soit l'auteur a réalisé son tableau à partir de ce poncif, soit il a effectué une excellente copie d'un tableau réalisé à partir de ce poncif.

Cependant, avant de mettre en évidence les éléments de la géométrie interne, il est nécessaire de définir ce maillage harmonique vertical : sa position et les dimensions de la maille.

\section{Le contour de la tête du Christ}

La nature présente peu de formes circulaires, l'anatomie humaine encore moins. Le contour de la tête du Christ n'est pas circulaire (figure 5), son crâne présente la forme d'un arc d'ellipse supérieur à $130^{\circ}$ (figure 6).

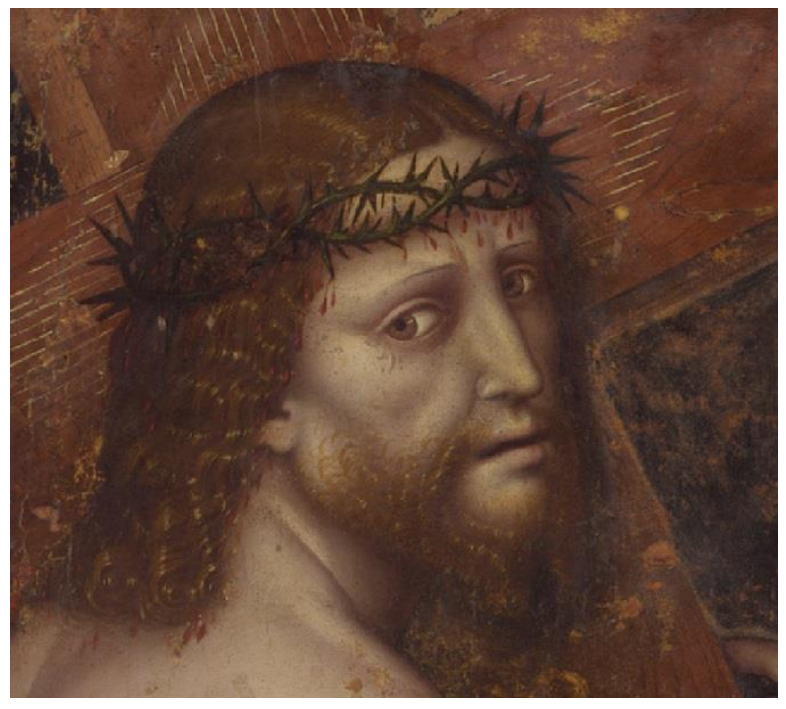

Figure 5. La tête du Christ.

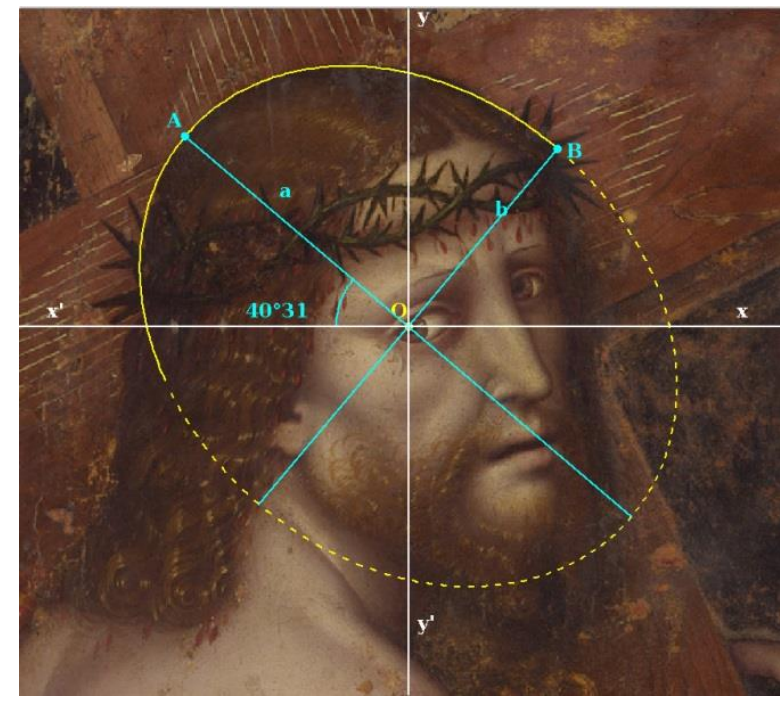

Figure 6. L'ellipse du contour de son crâne

L'ellipse est précisément centrée au point $\mathbf{O}$, au centre de la pupille de son œil droit. Elle a pour excentricité $\boldsymbol{\varepsilon}=\mathbf{f} / \mathbf{a}=\sqrt{3} / \sqrt{ } 8=0,612$. C'est, suivant notre nomenclature ${ }^{12}$, une ellipse de type 2 , faisant partie des ellipses ${ }^{13}$ que savait construire Léonard de Vinci. Ses trois paramètres : le demigrand axe $\mathbf{a}$, le demi-petit axe $\mathbf{b}$ et la distance focale $\mathbf{f}$, sont respectivement proportionnels à : $\sqrt{\mathbf{8}}, \sqrt{\mathbf{5}}$, $\sqrt{ }$ 3. Ils vérifient la relation propre à l'ellipse $: \mathbf{a}^{2}=\mathbf{b}^{\mathbf{2}}+\mathbf{f}^{2}$.Son grand axe est incliné de $40^{\circ} 31$, dont la tangente vaut $3 \sqrt{ } 2 / 5$.

\section{Discussion :}

Ce processus géométrique de la modélisation du contour de la tête d'un personnage tournée de trois-quarts, par un arc d'ellipse centrée sur la pupille de son œil, est très particulier.

\footnotetext{
12 J.-P. Crettez : [3], §. 2.4 .4

13 J.-P. Crettez : [5]
} 
Léonard de Vinci connaissait ce processus puisqu'il l'a appliqué dans deux portraits féminins ${ }^{14}$ : La Dame à l'hermine et la Joconde (figures 7 et 8). Pour le premier tableau, l'ellipse est centrée sur la pupille de l'œil droit de La Dame à l'hermine qui tourne sa tête vers la gauche, l'ellipse est inclinée de $54^{\circ} 74$, et elle a pour excentricité $\boldsymbol{\varepsilon}=\mathbf{f} / \mathbf{a}=1 / \sqrt{ } 2=0,707$; et pour le deuxième portrait, l'ellipse est centrée sur la pupille de l'œil gauche de de la Joconde qui tourne sa tête vers la droite, l'ellipse est placée verticalement, et elle a pour excentricité $\boldsymbol{\varepsilon}=\mathbf{f} / \mathbf{a}=0,5$.

Dans ces deux portraits, les excentricités et les inclinaisons sont différentes, mais dans les deux cas, le processus est le même. Le centre de l'ellipse, situé au centre de la pupille, est un point particulier qui coïncide avec un nœud du maillage. Ce point constitue un point important de la composition. L'axe vertical médian de la composition passe par ce point.

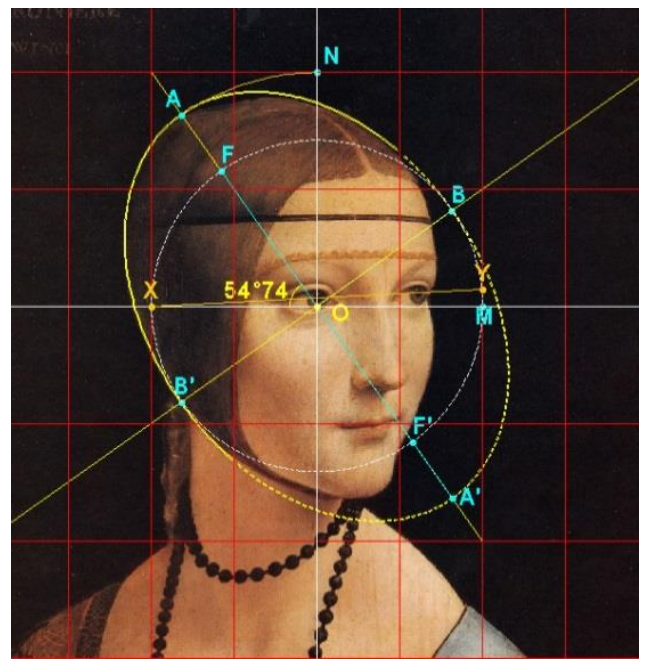

Figure 7. Contour du crâne de la Dame à l'hermine

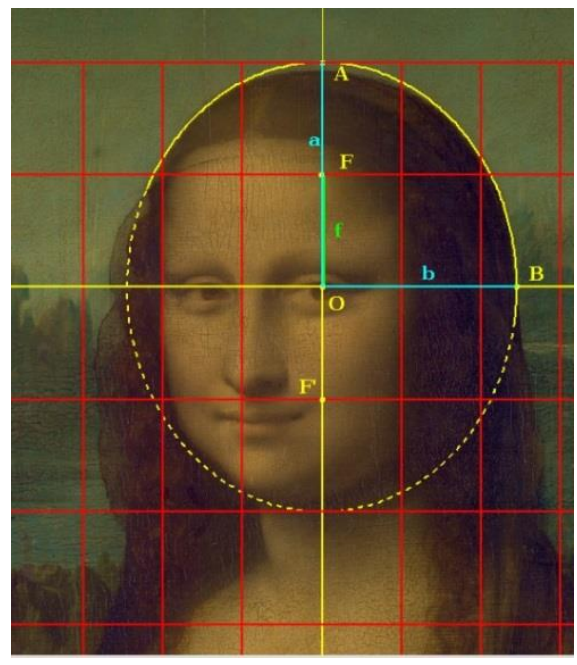

Figure 8. Contour du crâne de la Joconde

\section{Recherche du maillage}

L'auteur a probablement choisi d'appliquer le même processus pour établir la géométrie interne $d u$ Christ portant la croix, en plaçant (figure 9) le centre de l'ellipse $\mathbf{O}$ au centre de la pupille de son œil droit, et en faisant coïncider ce point avec un nœud du maillage.

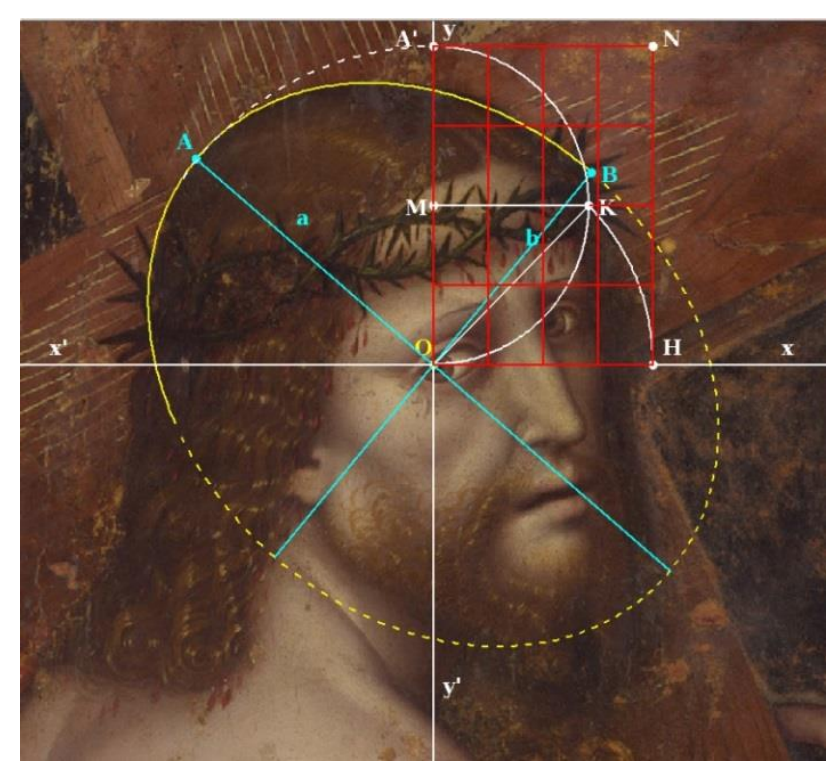

Figure 9. Maillage harmonique vertical partiel 
Soient $\mathbf{x} \mathbf{x}^{\prime}$ et $\mathbf{y} \mathbf{y}^{\prime}$ les axes de coordonnées passant par $\mathbf{O}$. Par rotation de centre $\mathbf{O}$ et de rayon $\mathbf{a}$, le segment $\mathbf{O A}$ se projette en $\mathbf{O A}^{\prime}$, avec $\mathbf{O A}^{\prime}=\mathbf{a}$ (figure 9). Soient $\mathbf{h}$ et $\mathbf{L}$ la hauteur et la largeur de la maille, avec la relation $\mathbf{h}=\mathbf{L} \sqrt{\mathbf{2}}$. Puisque le demi-grand axe a est proportionnel à $\sqrt{\mathbf{8}}$, et que $4=$ $\sqrt{2} . \sqrt{ } 8$, nous avons posé simplement $\mathbf{O A}^{\prime}=\mathbf{O A}=\mathbf{a}=4 \mathbf{h}$.

La largeur de la maille $\mathbf{L}$ se détermine géométriquement. Dans le demi-cercle de rayon $2 \mathbf{h}$, centré en $\mathbf{M}$ milieu de $\mathbf{O A}$ ', on trace la droite MK. Par rotation de centre $\mathbf{O}$ et de rayon $\mathbf{O K}=\mathbf{2 h} \sqrt{\mathbf{2}}$, le point $\mathbf{K}$ se projette en $\mathbf{H}$ avec $\mathbf{O H}=\mathbf{2} \mathbf{h} \sqrt{2}=4 \mathbf{L}$.

Le rectangle OA'NH de dimension ( $4 \mathbf{h} \times \mathbf{x} 4 \mathbf{L})$ est un rectangle harmonique. Sa subdivision $(4 \mathrm{x} 4)$ détermine (figure 8) un maillage harmonique vertical partiel.

\section{Détermination géométrique des paramètres de l'ellipse}

Par rotation le demi-grand axe $\mathbf{a}=\mathbf{O A}$ se projette sur l'axe vertical en $\mathbf{O A}^{\prime}$ '. Nous avons attribué à ce demi-grand axe la valeur $\mathbf{a}=4 \mathbf{h}=2 \mathbf{L} \sqrt{ } 8$.

L'arc de cercle de centre $\mathbf{O}$ et de rayon $4 \mathbf{L}$ coupe ce rectangle en $\mathbf{M}$ ', définissant le carré OM'N'H de côté $4 \mathbf{L}$ (figure 10). Le demi-petit axe b est égal à la diagonale $\mathbf{O B}$ ' du demi-carré. Il a pour valeur $\mathbf{b}=\mathbf{L} \sqrt{2} 0=2 \mathbf{L} \sqrt{5}$.

La distance focale $\mathbf{f}$ est égale à la diagonale d'un bloc de deux fois deux mailles $\mathbf{f}=\mathbf{O F}^{\prime}=\mathbf{L} \sqrt{ } 12$ $=2 \mathbf{L} \sqrt{ } 3$.

L'on vérifie que : $\mathbf{a}^{2}=\mathbf{b}^{2}+\mathbf{f}^{2}$. L'excentricité vaut $\boldsymbol{\varepsilon}=\mathbf{f} / \mathbf{a}=\sqrt{3} / \sqrt{ } 8=\mathbf{0 , 6 1 2}$.

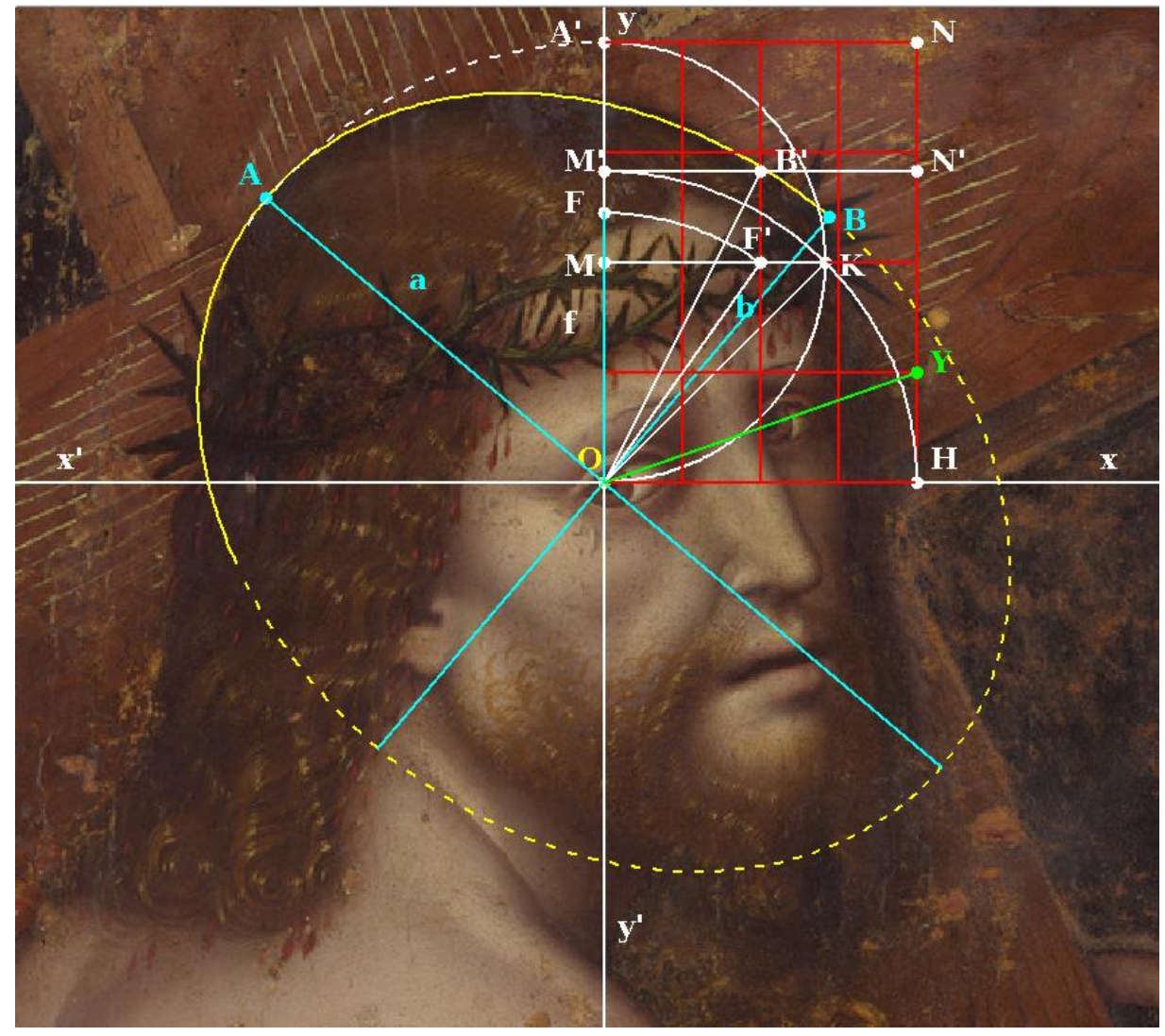

Figure 10. Calcul des paramètres $\boldsymbol{a}, \boldsymbol{b}, \boldsymbol{f}$ de l'ellipse du contour du crâne

\section{La ligne des yeux}

Le segment de droite $\mathbf{O Y}$, tracé sur le maillage, correspond à la ligne des yeux. Elle est inclinée d'un angle $\lambda=\mathbf{1 9}^{\circ} \mathbf{4 4}$, tel que $\operatorname{tg} \lambda=\mathbf{h} / 4 \mathbf{L}=\sqrt{2} / 4$. 


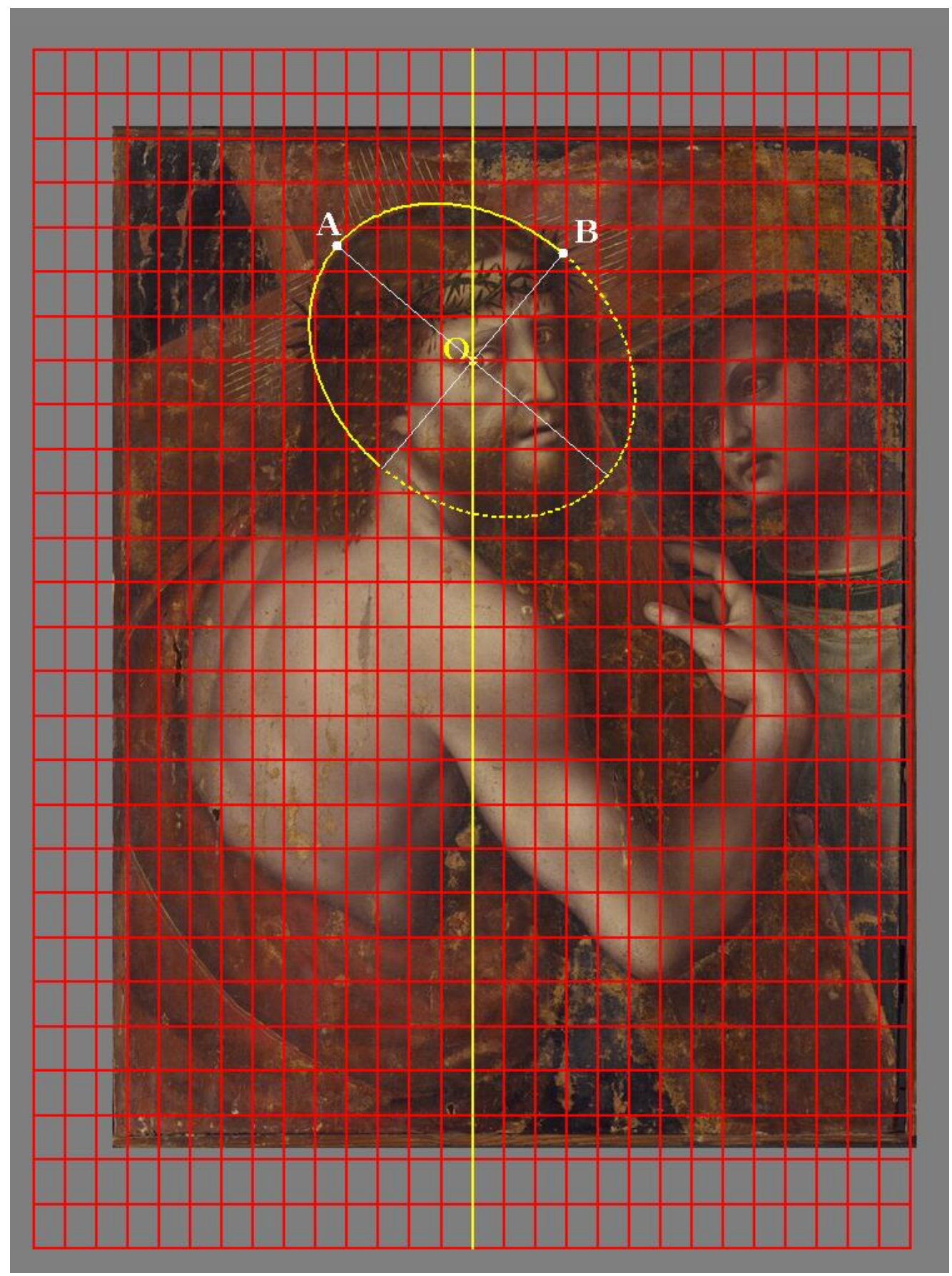

Figure 11. Extension du maillage à tout le tableau et au-delà

En prenant comme référence le point $\mathbf{O}$, centre de la pupille de l'œil droit du Christ, et en s'appuyant sur les paramètres de l'ellipse modélisant le crâne, il est maintenant possible de retrouver le maillage harmonique vertical (figure 11) qui a servi de support à la composition. La composition reposait sur un maillage de plus grande dimension, 28 lignes par 28 colonnes, formant ainsi un rectangle harmonique plus large et plus bas que le tableau, et permettant de recentrer le tableau et de dessiner une plus grande partie de la croix et de la tunique du Christ.

L'axe vertical médian de la composition passe par le point $\mathbf{O}$, centre de la pupille de l'œil droit, de la même façon que l'axe vertical médian passe par l'œil gauche de la Joconde (figure 12). Et comme dans la Joconde, l'œil est situé aux trois-quarts de la hauteur de la composition. 


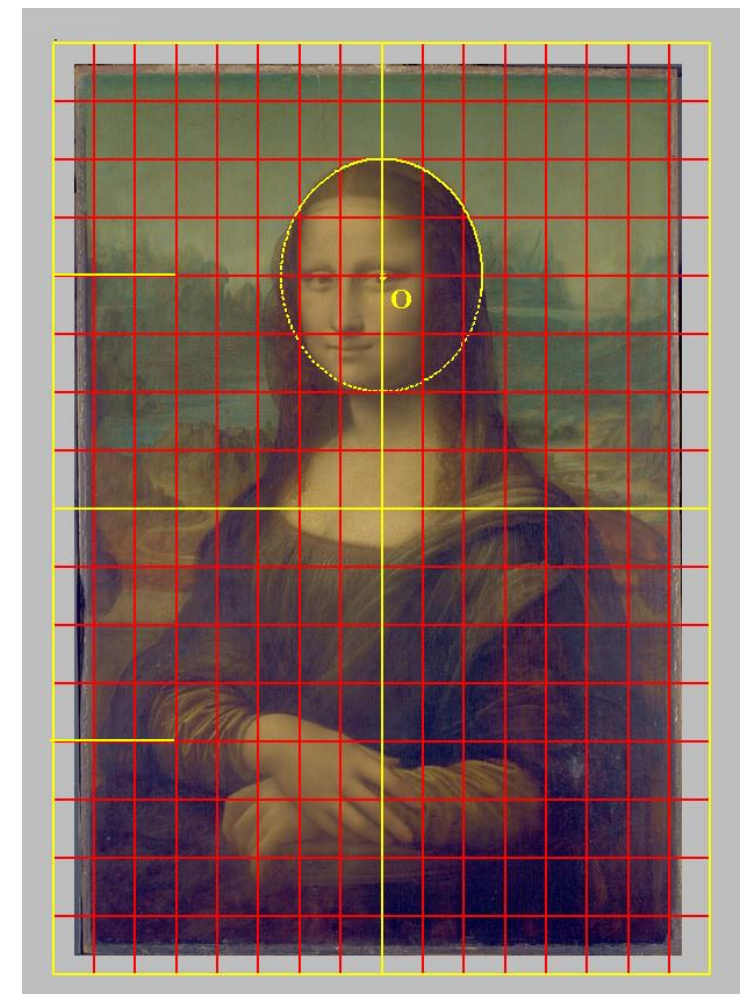

Figure 12. Le maillage de la Joconde

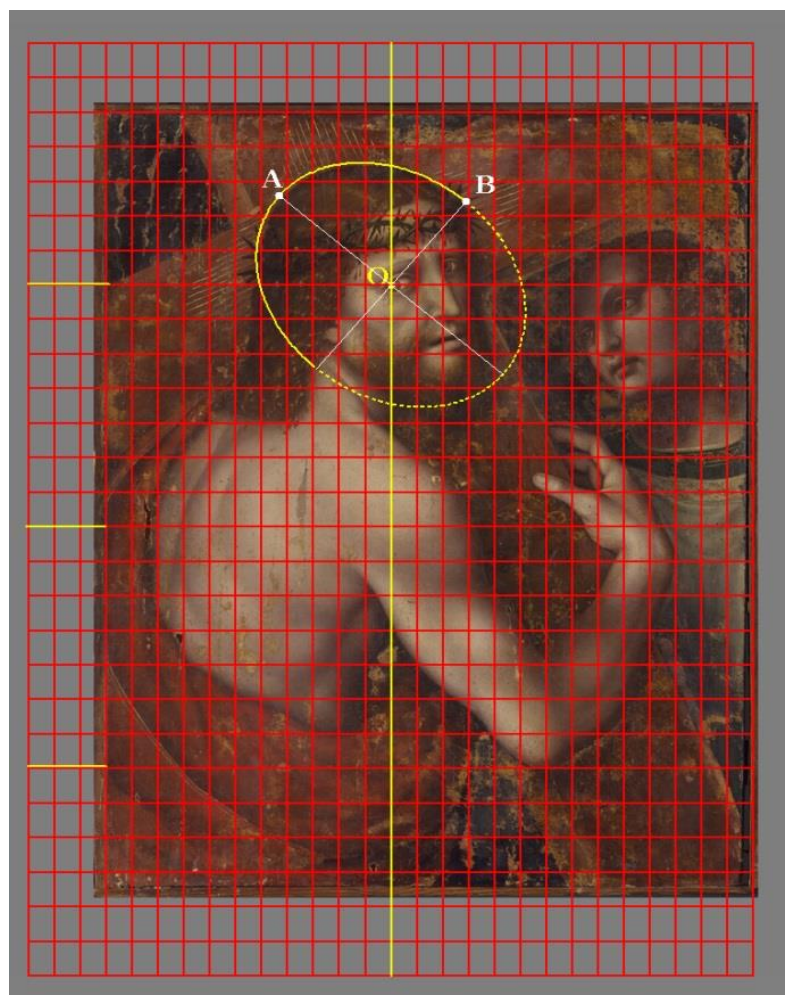

Figure 13. Le maillage du Christ portant sa croix

\section{Dimensions de la composition}

En se référant aux dimensions physiques du tableau, nous pouvons estimer, grâce à la quantification, que la composition devait avoir une largeur de l'ordre de 49,5 cm et une hauteur de $70,3 \mathrm{~cm}$.

La maille aurait pour hauteur $\mathbf{h}=2,51 \mathrm{~cm}$ et pour largeur $\mathbf{L}=1,78 \mathrm{~cm}$. La maille serait environ deux fois plus petite que celle du maillage de la Joconde.

\section{Tracé des bords de la croix}

Après avoir établi le maillage harmonique vertical, l'auteur a pu construire la géométrie interne de la composition. Il a commencé par dessiner les contours de la croix. Pour délimiter le montant, il a d'abord tracé (figure 14) deux lignes droites inclinées d'un angle $\boldsymbol{\alpha}$, en s'appuyant sur les nœuds du maillage (points bleus). Les nœuds successifs sont espacés de trois lignes et deux colonnes comme nous l'avons expliqué (figure 3). À cause de la régularité du maillage, ces deux lignes droites sont parallèles.

Puis pour délimiter les bras de la croix, il a tracé deux autres lignes droites inclinées d'un angle $\boldsymbol{\beta}$ en s'appuyant sur d'autres nœuds du maillage. Ces nœuds successifs sont espacés d'une ligne et de trois colonnes. Ces deux lignes droites sont parallèles. Mais grâce aux propriétés du maillage harmonique, ces deux droites sont orthogonales aux deux premières.

Bien plus, grâce aux nœuds du maillage harmonique, il a pu tracer les droites (en vert) montrant l'épaisseur du montant et des bras de la croix. Pour le montant, la droite est située deux lignes plus bas que l'arête, et pour les bras la ligne est située une colonne à droite.

La largeur du montant est égale à 6,33 $\mathbf{L}$ et celle des bras est inférieure et égale à 5,95 $\mathbf{L}$ 
Comme on peut le constater, le processus utilisé par l'auteur pour tracer ces droites est simple, précis et convaincant. Il ne nécessite aucun calcul, aucun appareil de mesure.

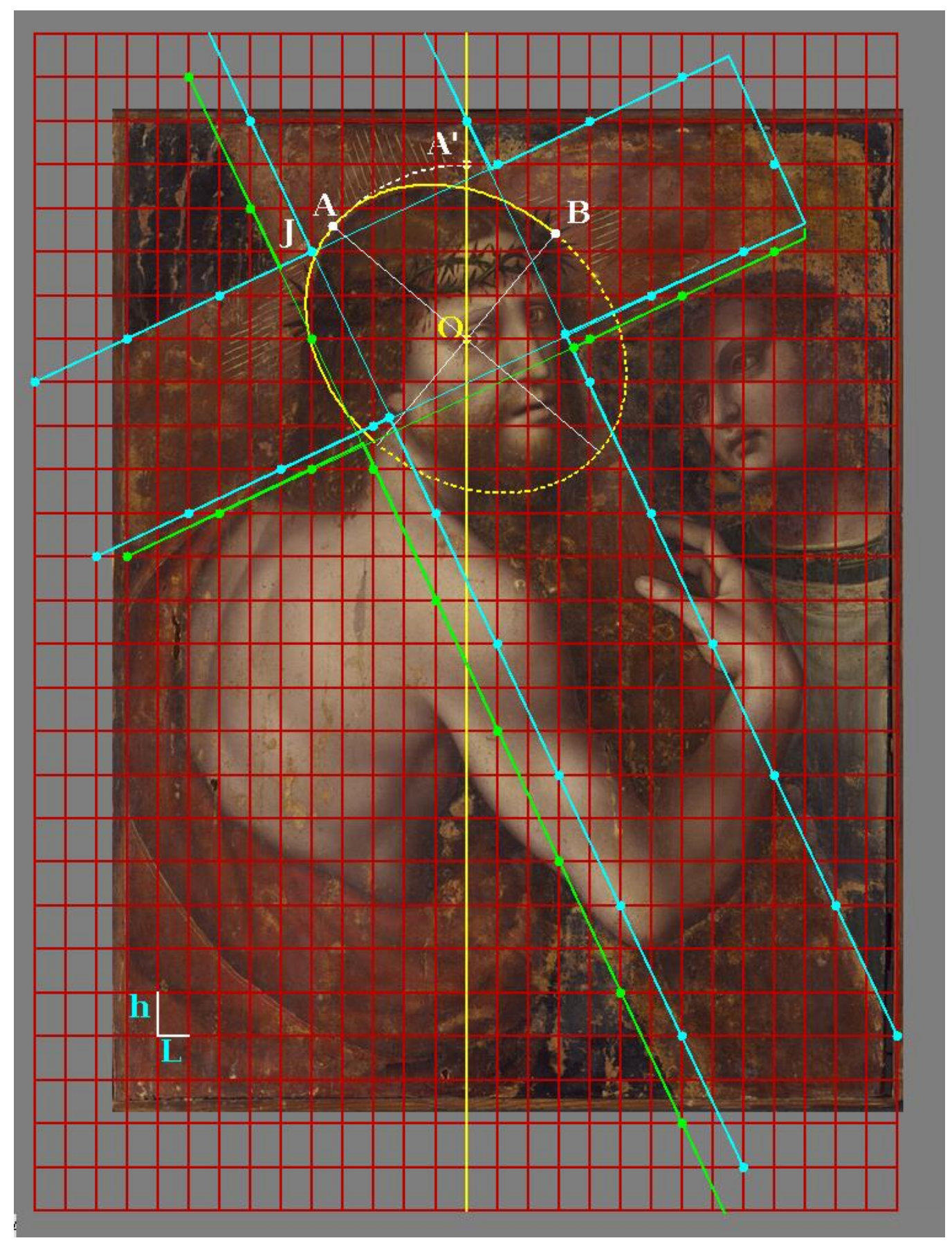

Figure 14. Le tracé des bords des pièces de bois formant la croix

\section{Les plis de la tunique du Christ}

Le peintre a brossé la tunique du Christ avec de nombreux plis, comme une draperie. La tunique glisse vers le bas de façon naturelle, en découvrant son dos nu et en formant deux grands plis plus prononcés.

L'auteur a donné au premier grand pli (figure 15) la forme d'un arc d'ellipse de plus de $160^{\circ}$. L'ellipse est centrée sur l'axe vertical médian, au point $\mathbf{O}^{\prime}, 7$ mailles plus bas que le centre $\mathbf{O}$. Son grand axe, incliné d'un angle $\beta=25^{\circ} 24$, est parallèle aux bras de la croix. Elle a la même excentricité $\varepsilon=0,612$ que l'ellipse qui entoure la tête du Christ. 


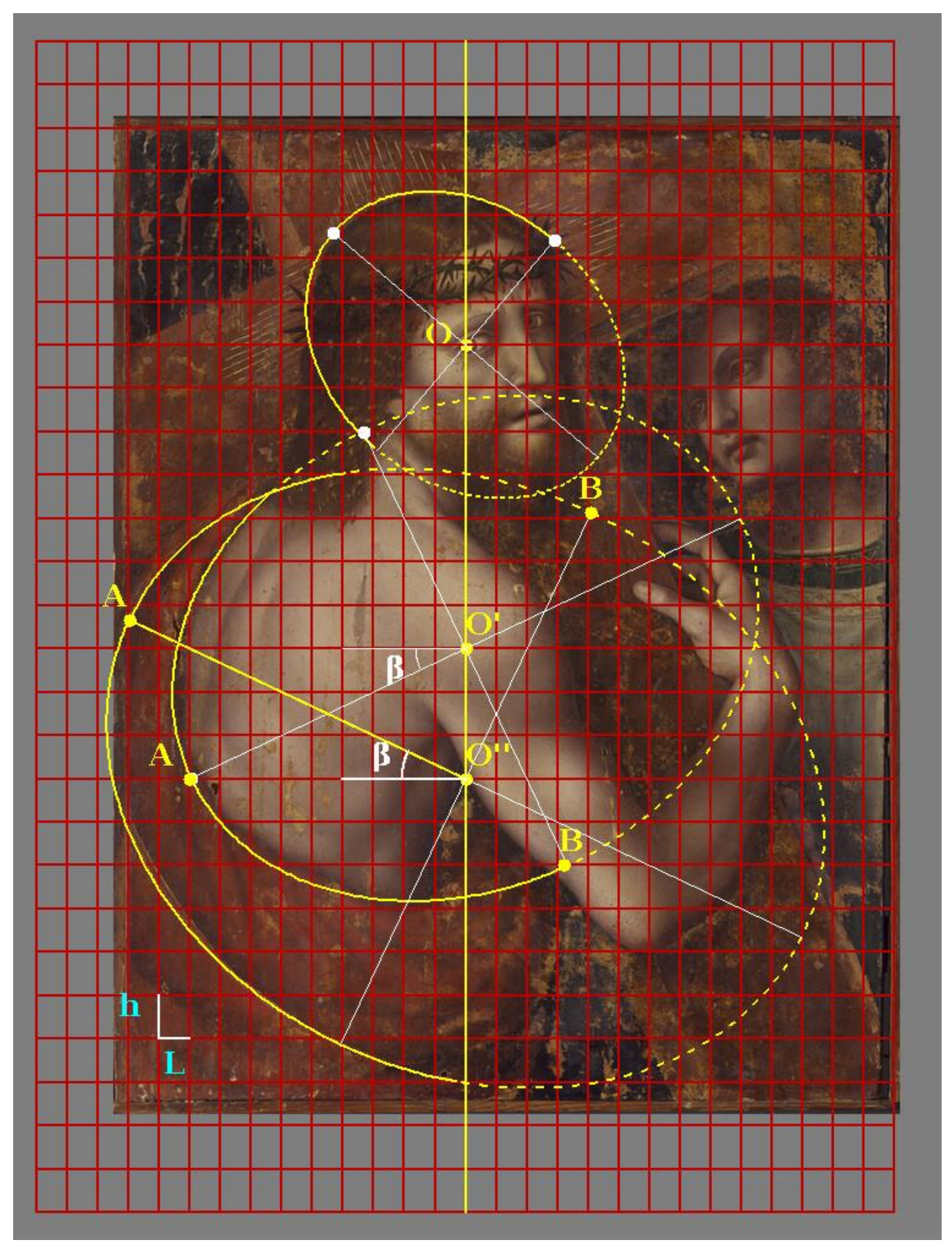

Figure 15. Les deux plis de la tunique

Le deuxième grand pli (figure 15) est plus important. Il peut également être modélisé (figure 15) par un arc d'ellipse de plus de $100^{\circ}$. L'ellipse est également centrée sur l'axe vertical médian, au point $\mathbf{O}^{\prime}$ ', 3 mailles plus bas que le centre $\mathbf{O}^{\prime}$. Son grand axe est incliné d'un angle $\boldsymbol{\beta}=-25^{\circ} 24$, symétriquement par rapport à celui de la première ellipse, à la recherche d'un certain équilibre. Cette ellipse possède aussi la même excentricité : $\varepsilon=0,612$. C'est encore une ellipse de type 2.

Nous allons maintenant apporter plus de précision sur ces deux arcs d'ellipse. 
Détermination géométrique des paramètres de l'ellipse du premier pli de la tunique

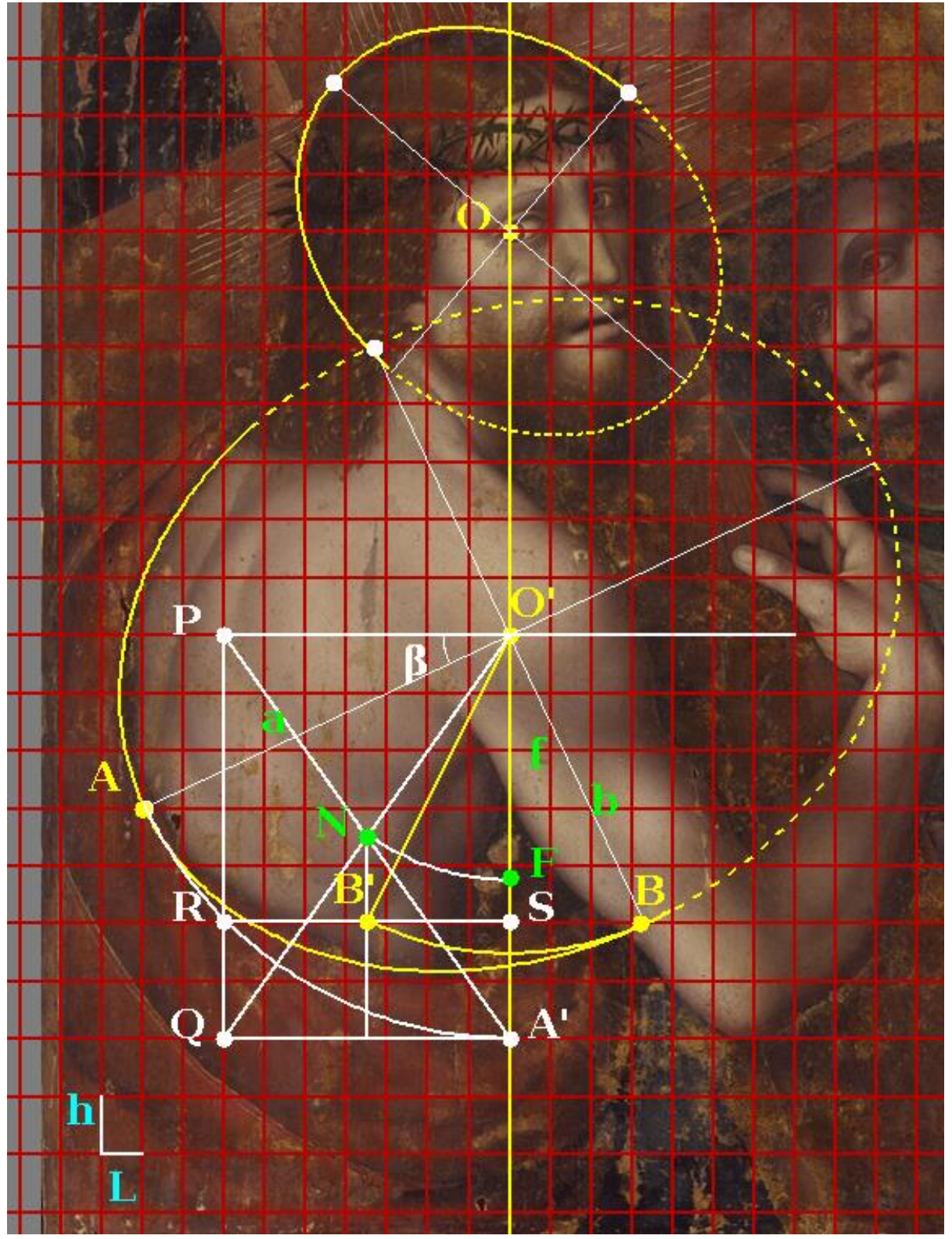

Figure 16. Détermination géométrique des paramètres de l'ellipse du premier pli de la tunique

Par rotation, le demi-grand axe $\mathbf{a}=\mathbf{O}^{\prime} \mathbf{A}$ se projette sur l'axe vertical en $\mathbf{O}^{\prime} \mathbf{A}$ '. L'auteur a donné à ce demi-grand axe la valeur $\mathbf{a}=7 \mathbf{h}$.

Pour déterminer les deux autres paramètres, on peut tracer, en s'appuyant sur $\mathbf{O}^{\prime} \mathbf{A}^{\prime}$, le rectangle harmonique O'PQA' de 7 fois 7 mailles, soit $7 \mathbf{h} \times \mathbf{7 L}$. L'arc de cercle de centre $\mathbf{O}$ ' et de rayon a, coupe ce rectangle en $\mathbf{R}$, définissant le carré $\mathbf{O}^{\prime} \mathbf{P R S}$ de côté $7 \mathbf{L}$. Le demi-petit axe b est égal à la diagonale O'B' du demi-carré. Il a pour valeur $\mathbf{b}=(7 \mathbf{L} / 2) \sqrt{5}=7 \mathbf{h} \sqrt{ } 5 / \sqrt{ } 8=\mathbf{a} \sqrt{5} / \sqrt{ } 8$.

La distance focale $\mathbf{f}=\mathbf{O}^{\prime} \mathbf{F}=\mathbf{O}^{\prime} \mathbf{N}=\mathbf{O}^{\prime} \mathbf{Q} / 2=7 \mathbf{L} \sqrt{ } 3 / 2=7 \mathbf{h} \sqrt{ } 3 / \sqrt{ } 8=\mathbf{a} \sqrt{ } 3 / \sqrt{ } 8$.

L'on vérifie que : $\mathbf{a}^{2}=\mathbf{b}^{2}+\mathbf{f}^{2}$. L'excentricité vaut $\boldsymbol{\varepsilon}=\mathbf{f} / \mathbf{a}=\sqrt{ } 3 / \sqrt{ } 8=\mathbf{0 , 6 1 2}$. 


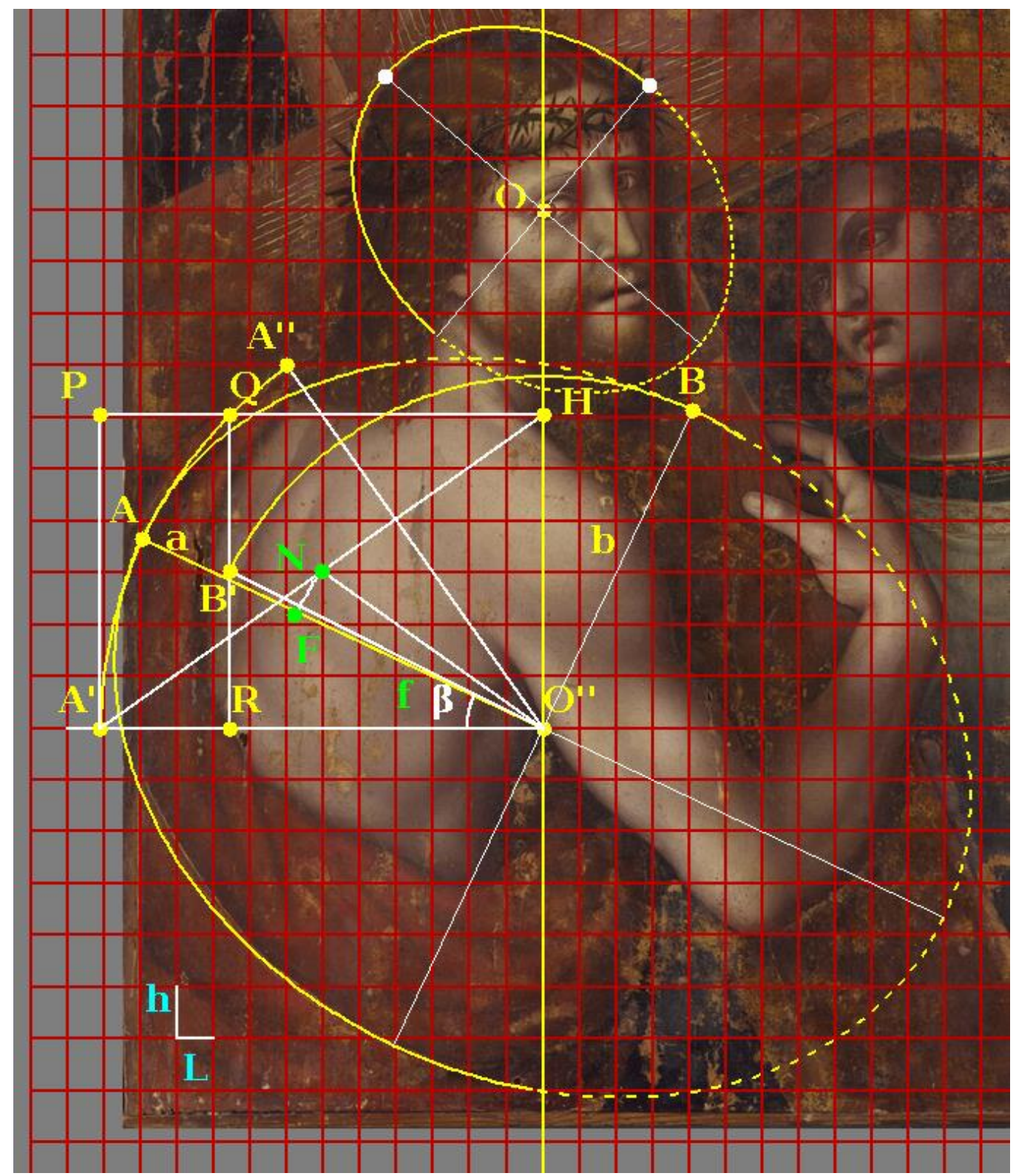

Figure 17. Détermination géométrique des paramètres de l'ellipse du deuxième pli de la tunique

L'auteur a donné au demi-grand axe O'A la valeur du segment O'A' qui mesure 7 diagonales de maille, soit $\mathbf{a}=7 \mathbf{L} \sqrt{ } 3$ (figure 17).

Par rotation, le segment O'A vient en O'A'. Sur ce segment $\mathbf{O}$ ''A' nous construisons comme précédemment le rectangle harmonique $\mathbf{O}$ ''A'PH dont le petit côté vaut $7 \mathbf{L} \sqrt{ } 3 / \sqrt{ } 2$. L'arc de cercle de centre $\mathbf{O}$ ' et de rayon a coupe ce rectangle au point $\mathbf{Q}$, définissant le carré $\mathbf{O}$ ' $\mathbf{H Q R}$ de côté $7 \mathbf{L} \sqrt{ } 3 / \sqrt{ } 2$. Le demi-petit axe $\mathbf{b}$ est égal à la diagonale $\mathbf{O}$ '' $\mathbf{B}$ ' du demi-carré. Il a pour valeur $\mathbf{b}=$ $(7 \mathbf{L} \sqrt{ } 3 / \sqrt{ } 8) \sqrt{ } 5)=\mathbf{a} \sqrt{ } 5 / \sqrt{ } 8$. La distance focale $\mathbf{f}=\mathbf{O}{ }^{\prime} \mathbf{F}=\mathbf{O}^{\prime}{ }^{\prime} \mathbf{N}=\mathbf{O}{ }^{\prime} \mathbf{P} / 2=(7 \mathbf{L} \sqrt{ } 3 / \sqrt{ } 2) \sqrt{ } 3 / 2=\mathbf{a} \sqrt{ } 3 / \sqrt{ } 8$.

L'on vérifie que : $\mathbf{a}^{2}=\mathbf{b}^{2}+\mathbf{f}^{2}$. L'excentricité vaut $\boldsymbol{\varepsilon}=\mathbf{f} / \mathbf{a}=\sqrt{ } 3 / \sqrt{ } 8=\mathbf{0 , 6 1 2}$.

Puisque $\mathbf{a}=7 \mathbf{L} \sqrt{ } 3=7 \mathbf{h} \sqrt{ }(3 / 2)$, cette deuxième ellipse est $\sqrt{ }(3 / 2)$ fois plus grande que la première. Le rapport de la surface de la première sur la surface de la seconde est égal à 2/3. 


\section{Propriété des deux ellipses des plis de la tunique du Christ}

Les deux ellipses des plis de la tunique sont centrées sur l'axe vertical médian, orientées symétriquement, elles ont même excentricité $\boldsymbol{\varepsilon}=\mathbf{0 , 6 1 2}$. Elles sont visuellement consonantes et puisque le rapport de leur surface est égal à $2 / 3$, elles résonnent à la quinte. Cette conception de deux ellipses consonantes se retrouve dans la Joconde ${ }^{15}$, où l'ellipse du visage et celle du voile sont consonantes entre elles et résonnent à l'octave ; mais aussi dans le Salvator Mundi ${ }^{16}$, où l'ellipse de la tête et celle du visage, sont consonantes entre elles et résonnent aussi à l'octave.

\section{Géométrie interne du Christ portant sa croix (Giampietrino National Gallery, Londres)}

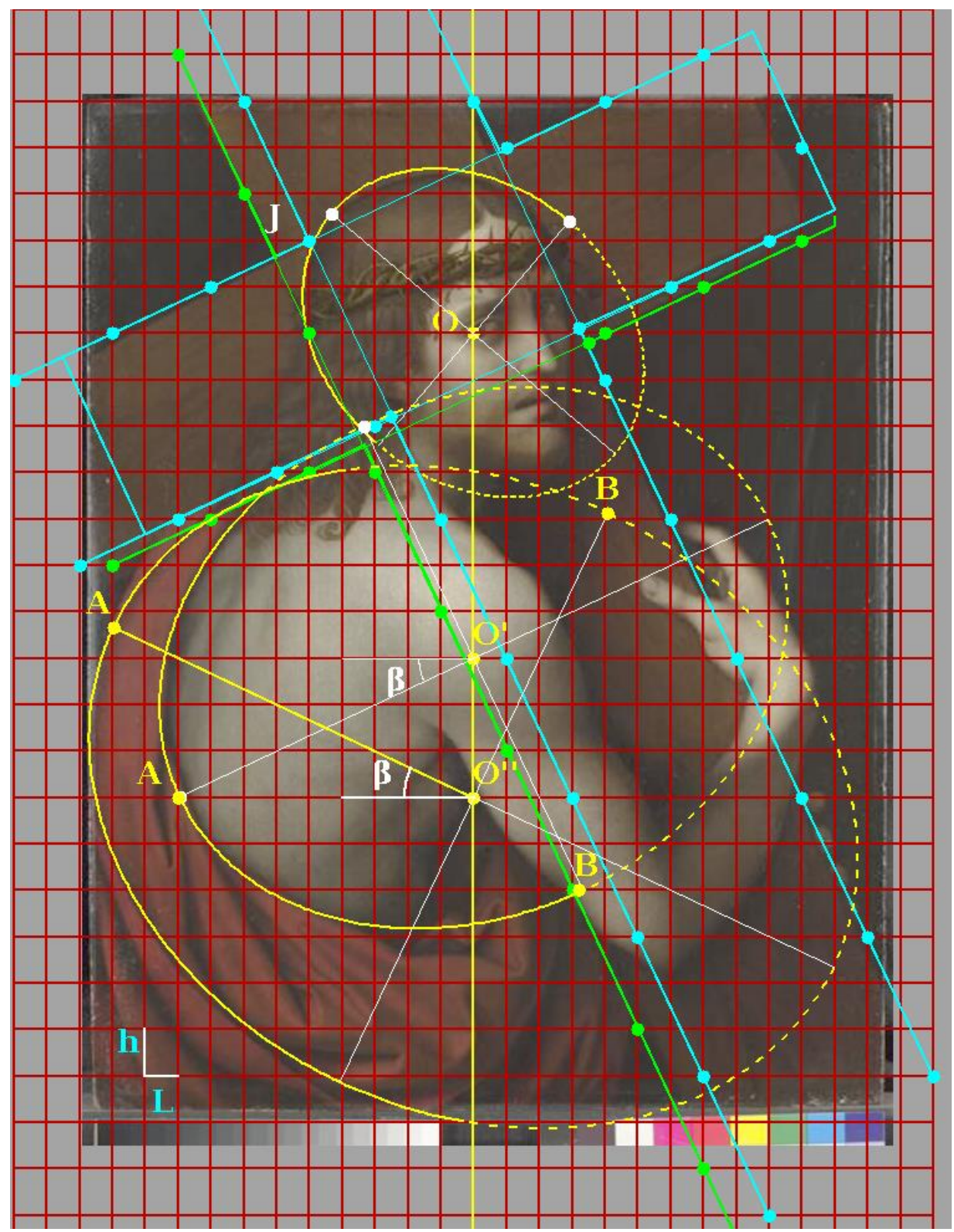

Figure 18. Géométrie interne du Christ portant sa croix, version National Gallery (Londres)

Nous avons appliqué à la version de la National Gallery, le même algorithme que celui qui nous a permis d'analyser la version exposée ci-dessus. Les deux versions présentent la même géométrie

\footnotetext{
${ }^{15}$ J-P. Crettez: [3], §. 8.2.3.5

${ }^{16}$ J-P. Crettez: [6]
} 
interne (figures 15 et 18). Les deux versions semblent donc avoir été tracées à partir du même poncif.

\section{Géométrie interne du Christ portant sa croix (Musée des Beaux-Arts, Budapest)}

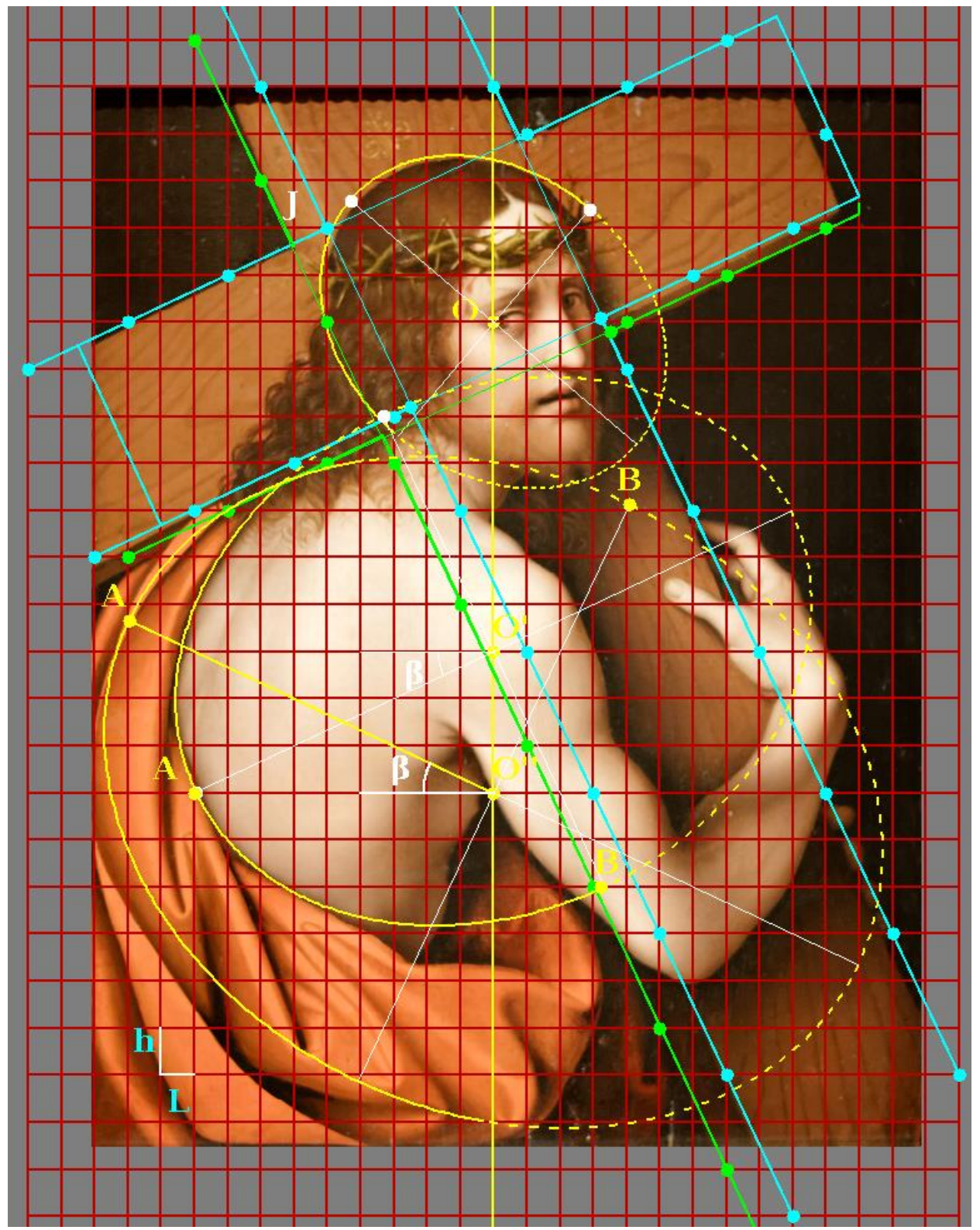

Figure 19. Géométrie interne du Christ portant sa croix, version du Musée des Beaux-Arts Budapest

Le même algorithme appliqué à la version de Budapest, montre un accord entre les deux versions seulement dans la partie haute (figure 19). L'écart avec le deuxième pli est manifeste. L'auteur de cette version n'a pas respecté la consonance visuelle.

\section{Conclusion}

Une simple observation de la valeur de l'angle d'inclinaison de la croix montre que le Christ portant la croix a été tracé à l'aide d'un poncif provenant d'une composition établie à partir d'une géométrie interne basée sur un maillage harmonique vertical.

Le maillage harmonique, indispensable au tracé de la géométrie interne, a permis à l'auteur de tracer avec précision les bois de la croix, leur orientation particulière, leur parallélisme, mais aussi de représenter leur épaisseur. 
Grâce au maillage harmonique, l'auteur a pu calculer, orienter et tracer les formes elliptiques en leur conférant une même excentricité. En particulier il a su modéliser le contour du crâne du Christ, avec une ellipse centrée sur la pupille de son œil droit. Par ce point passe l'axe vertical médian de la composition sur lequel sont centrées les deux autres ellipses. Cet œil (fenêtre de l'âme) est assurément le point le plus «sensible » de la composition. Ce processus consistant à centrer l'ellipse modélisant le contour du crâne du personnage principal est très léonardesque puisque nous le retrouvons dans la Joconde et dans la Dame à l'hermine.

Ensuite, l'auteur a dessiné la tunique du Christ avec de nombreux plis comme une draperie : spécialité de Léonard de Vinci. Bien plus, il a cherché à modéliser les deux grands plis de la tunique en leur conférant deux formes elliptiques consonantes suivant un principe souvent recherché par Léonard.

Ces quelques observations géométriques nous incitent à considérer que le poncif (issu de la géométrie interne), qui a servi à la construction de cette version du Christ portant la croix et probablement à celle des autres versions (en totalité ou en partie), semble avoir été effectué par l'auteur de la Joconde.

\section{Bibliographie}

[1] Bouleau Ch., Charpentes : La géométrie secrète des peintres, Le Seuil, Paris, 1963.

[2] Clark K., Léonard de Vinci, Librairie générale Française, Paris, 2008.

[3] Crettez J.-P., Les supports de la géométrie interne des peintres : de Cimabue à G. de La Tour. Éditions ISTE (2017).

[4] Crettez J.-P., Openscience - Arts-et-Sciences - Géométrie interne d'une "Nuit" de G. de La Tour : "L’Apparition de l'ange à saint Joseph" (musée des beaux-arts, Nantes), Openscience - Arts-et-Sciences 2018, Vol. 1, n 1. https://www.openscience.fr/IMG/pdf/iste_artsci18v1n1.pdf

[5] Crettez J.-P., Openscience - Léonard de Vinci et le tracé des formes elliptiques. Openscience - Arts-et-Sciences 2021, Vol. 5, n². http://www.openscience.fr/IMG/pdf/iste_artsci21v5n2_2.pdf

[6] Crettez J.-P., Openscience - Géométrie interne du «Salvator Mundi». Openscience- Arts-et-Sciences 2019, Vol. 3, $\mathrm{n}^{\circ} 1$. https://www.openscience.fr/IMG/pdf/iste_artsci19v3n1_3.pdf

[7] Léonard de Vinci, Le traité de peinture, note 354, p.149, Éditions Jean de Bonnot, Paris 1982. 